\title{
Actin polymerization state regulates osteogenic differentiation in human adipose-derived stem cells
}

\author{
Bing Sun ${ }^{1 \dagger}$, Rongmei Qu ${ }^{1 \dagger}$, Tingyu Fan ${ }^{1 \dagger}$, Yuchao Yang ${ }^{1}$, Xin Jiang ${ }^{1}$, Asmat Ullah Khan ${ }^{1}$, Zhitao Zhou², \\ Jingliao Zhang ${ }^{3}$, Kuanhai Wei ${ }^{4}$, Jun Ouyang ${ }^{1 *}$ and Jingxing Dai ${ }^{i^{*}}$ (D
}

*Correspondence:
jouyang@smu.edu.cn;
daijx@smu.edu.cn
${ }^{\dagger}$ Bing Sun, Rongmei Qu,
and Tingyu Fan contributed
equally to this article
${ }^{1}$ Guangdong Provincial
Key Laboratory
of Medical Biomechanics
and Department of Anatomy,
School of Basic Medical
Science, Southern Medical
University, Guangzhou, China
Full list of author information
is available at the end of the
article

${ }^{*}$ Correspondence: jouyang@smu.edu.cn

${ }^{\dagger}$ Bing Sun, Rongmei Qu, and Tingyu Fan contributed equally to this article

gdong Provincial

and Department of Anatomy School of Basic Medical University, Guangzhou, Chin is available at the end of the article

\begin{abstract}
Background: Actin is an essential cellular protein that assembles into microfilaments and regulates numerous processes such as cell migration, maintenance of cell shape, and material transport.

Methods: In this study, we explored the effect of actin polymerization state on the osteogenic differentiation of human adipose-derived stem cells (hASCs). The hASCs were treated for 7 days with different concentrations $(0,1,5,10,20$, and $50 \mathrm{nM})$ of jasplakinolide (JAS), a reagent that directly polymerizes F-actin. The effects of the actin polymerization state on cell proliferation, apoptosis, migration, and the maturity of focal adhesion-related proteins were assessed. In addition, western blotting and alizarin red staining assays were performed to assess osteogenic differentiation.
\end{abstract}

Results: Cell proliferation and migration in the JAS $(0,1,5,10$, and $20 \mathrm{nM})$ groups were higher than in the control group and the JAS (50 nM) group. The FAK, vinculin, paxillin, and talin protein expression levels were highest in the JAS (20 nM) group, while zyxin expression was highest in the JAS $(50 \mathrm{nM})$ group. Western blotting showed that osteogenic differentiation in the JAS $(0,1,5,10,20$, and $50 \mathrm{nM})$ group was enhanced compared with that in the control group, and was strongest in the JAS (50 nM) group.

Conclusions: In summary, our data suggest that the actin polymerization state may promote the osteogenic differentiation of hASCs by regulating the protein expression of focal adhesion-associated proteins in a concentration-dependent manner. Our findings provide valuable information for exploring the mechanism of osteogenic differentiation in hASCs.

Keywords: Human adipose-derived stem cells, Actin polymerization, Osteogenic differentiation, Jasplakinolide, Cell proliferation and migration

\section{Background}

Actin, a single polypeptide chain globular protein with a molecular weight of $42 \mathrm{kDa}$, is the primary component of the cytoskeleton. There are two main forms of actin, the globular actin monomers (G-actin) and the polymeric actin filaments (F-actin). Actin plays a crucial role in many biological systems. The polymerization/depolymerization author(s) and the source, provide a link to the Creative Commons licence, and indicate if changes were made. The images or other third party material in this article are included in the article's Creative Commons licence, unless indicated otherwise in a credit line to the material. If material is not included in the article's Creative Commons licence and your intended use is not permitted by statutory regulation or exceeds the permitted use, you will need to obtain permission directly from the copyright holder. To view a copy of this licence, visit http:// creativecommons.org/licenses/by/4.0/. 
of actin is closely related to numerous cell activities, including the maintenance of cell morphology [2], cell movement [3], cytoplasmic streaming [3], material transport, apical growth, physical, and chemical signal transduction [4-7]. The dynamic remodeling of F-actin plays a crucial role in many diseases, such as cancer $[8,9]$ and malaria [10-12], and is additionally involved in the wound healing process, embryonic development, and tissue formation [13, 14].

In recent decades, several substances involved in the regulation of actin polymerization and depolymerization have been investigated. These include phalloidin [15], cytochalasin D [16], and latrunculin A $[17,18]$, which can alter intracellular actin organization [19]. This group also includes jasplakinolide/jaspamide (JAS) [20-25], which was originally isolated from a marine sponge [20, 25-27]. A major advantage of JAS is that it is membrane permeable [28], which makes it an ideal tool for stabilizing or polymerizing actin filaments in live cells. It induces the nucleation of actin filaments, reduces the rate of dissociation of actin subunits from F-actin filaments, and prevents cofilin from severing F-actin filaments, resulting in filament stabilization [27, 29, 30].

Adipose-derived stem cells (ASCs) are a type of adult mesenchymal stem cell derived from fat tissue. They self-replicate and can differentiate in multiple directions [31-34], including the osteogenic, adipogenic [33, 35, 36], chondrogenic [37], and myogenic $[36,38]$ routes. ASCs are a good cell source for stem cell research [31] because they are widely available, easy to isolate, proliferate stably in vitro, and have a low decay rate $[33,39,40]$. Numerous studies have demonstrated that the polymerization of the actin cytoskeleton can affect cell migration, proliferation, and differentiation [3, 41]. Recent studies have shown that actin microfilament expression increased during the process of osteogenic differentiation. These microfilaments are orderly, thick, and arranged in a filamentous shape [42, 43]. However, how osteogenic differentiation is affected by the extent of actin filament polymerization remains unclear. Therefore, in our study, human ASCs (hASCs) were treated with different concentrations of JAS (resulting in different degrees of polymerization) to study the influence of actin polymerization state on the osteogenic differentiation of hASCs.

\section{Materials and methods}

\section{Cell culture and jasplakinolide assay}

After obtaining informed consent from all patients and approval from the Southern Medical University (Guangzhou, China) ethics committee, leftover subcutaneous adipose tissue was acquired from patients undergoing orthopedic surgery. Human fat tissue was washed with phosphate-buffered saline (PBS) three times, cut into pieces, and then incubated in $0.15 \%$ collagenase type I (Sigma, NY, USA) at $37^{\circ} \mathrm{C}, 5 \% \mathrm{CO}_{2}$ for $60 \mathrm{~min}$. An equal volume of growth medium containing 10\% FBS (Gibco, NY, USA) and 1\% Penicillin-Streptomycin (Gibco, NY, USA) was used to terminate digestion. This mixture was centrifuged for $5 \mathrm{~min}$ at $250 \mathrm{~g}$ (room temperature), the supernatant was removed, and the pellet was suspended in growth medium and incubated at $37{ }^{\circ} \mathrm{C}, 5 \% \mathrm{CO}_{2}$. After $24 \mathrm{~h}$, the medium was completely replaced with fresh medium, and subsequently changed every other day. The cells were passaged at $80 \%$ confluence and used between the 3rd and 5th passage (plating density $=6000 \mathrm{cells} / \mathrm{cm}^{2}$ ). After $24 \mathrm{~h}$, cells were treated daily with a growth medium or osteogenic differentiation medium containing different 
concentrations of jasplakinolide (JAS), or control medium containing the same volume of DMSO. The osteogenic differentiation medium (OS) consisted of DMEM supplemented with 10\% FBS (Gibco, NY, USA), 1\% Penicillin-Streptomycin (Gibco, NY, USA), $10 \mathrm{mM} \beta$-glycerophosphate (Sigma, NY, USA), $100 \mathrm{nM}$ dexamethasone and $37.5 \mathrm{mg} / \mathrm{L}$ ascorbic acid (Sigma, NY, USA).

\section{Cell Counting Kit-8 (CCK-8) assay}

Discarding the medium, cells were incubated with CCK-8 solution (Dojindo Cell Counting Kit-8) according to the manufacturer's instructions $\left(37^{\circ} \mathrm{C}, 5 \% \mathrm{CO}_{2}\right.$ incubator). After $0.5 \mathrm{~h}$, absorbance was measured at $450 \mathrm{~nm}$ using a microplate reader (Bio-Rad, USA).

\section{Immunofluorescence assay}

Cells were washed two or three times with PBS, then fixed in $4 \%$ paraformaldehyde for $10 \mathrm{~min}$, washed three times with PBS, and permeabilized with $0.1 \%$ Triton X-100 for $5 \mathrm{~min}$, and blocked in 2\% BSA for $1 \mathrm{~h}$ at room temperature. Next, cells were incubated with primary antibodies (YAP, 1:700, Cell Signaling Technology, USA; Ki67, 1:500, Abcam, USA) overnight at $4{ }^{\circ} \mathrm{C}$. The corresponding secondary antibody was then added to samples and incubated at room temperature for $1 \mathrm{~h}$. These antibodies included Cy3 labeled goat anti-rabbit IgG $(\mathrm{H}+\mathrm{L})$, Beyotime, China; Alexa Fluor 546 goat anti-mouse, Life Technologies, USA; or Alexa Fluor 488 phalloidin, Thermo Fisher Scientific, USA. After washing three times with PBS, cells were incubated with DAPI. The images were observed and captured using a fluorescence microscope (Carl Zeiss; German). Data analysis was performed using Image $1.52 \mathrm{~V}$ (NIH, USA).

\section{Cell apoptosis}

Cells were washed three times with PBS and digested with trypsin (without EDTA). Then cells were recovered by centrifugation ( $500 \mathrm{~g}$ for $5 \mathrm{~min}$ ), washed with pre-cooled PBS, and then centrifuged ( $500 \mathrm{~g}$ for $5 \mathrm{~min}$ ) again (this process was repeated before proceeding). To measure apoptosis, we used the Annexin V-FITC/Propidium Iodide (PI) Apoptosis Detection Kit (Dalian Meilun Biotechnology, China). We added appropriate volumes of working solution to the cell pellet, resuspended the cells, and reconstituted the cells at a density of $1 \times 10^{6}$ cells $/ \mathrm{mL}$. Next, we pipetted $100 \mu \mathrm{L}$ of cell suspension $\left(1 \times 10^{5}\right.$ cells) into a new tube, added both Annexin V-FITC and PI $(5 \mu \mathrm{L})$, and incubated at room temperature for $15 \mathrm{~min}$. Lastly, $400 \mu \mathrm{L}$ of the working solution was added to each tube, and the level of fluorescence was detected by flow cytometry (BD LSR Fortessa X-20, BD, USA). FlowJo 7.6.1 software was used to analyze the data Q2+Q3.

\section{Wound healing assay}

The cells were plated on a fibronectin-coated 6-well plate. After complete attachment, a vertical scratch was made at the bottom of the well with a $10 \mu \mathrm{L}$ pipette tip. After washing the cells three times with PBS, a complete medium containing different concentrations of JAS was added to each well. The cells were cultured in an incubator at $37^{\circ} \mathrm{C}$, under $5 \% \mathrm{CO}_{2}$. We observed cell migration at different time points $(0,6,12$, and $24 \mathrm{~h})$. According to the observations, the results at other time points were consistent with the trend at $24 \mathrm{~h}$, so we have only shown images at $0 \mathrm{~h}$ and $24 \mathrm{~h}$ here. Images of cells were 
collected using an inverted microscope (1MT-2-21, Olympus, Japan) and the wound area at 0 and $24 \mathrm{~h}$ was quantified with the ImageJ software (ImageJ 1.46r, Wayne Rasband National Institutes of Health, USA).

\section{Transwell assay}

We suspended the cells in growth medium without FBS, added $100 \mu \mathrm{L}$ of the cell suspension to the upper chamber, and then added medium (20\% FBS) with different concentrations of JAS to the lower chamber. After $24 \mathrm{~h}$, cells were fixed in $4 \%$ paraformaldehyde for $10 \mathrm{~min}$ and stained with $0.1 \%$ crystal violet (crystal violet-citric acid staining solution, Soleil, China) for $30 \mathrm{~min}$. Images were obtained with an Olympus microscope (1MT-2-21, Olympus, Japan).

\section{Alkaline phosphatase activity}

Cells were washed three times with PBS and permeabilized with $1 \%$ Triton X-100 for $30 \mathrm{~min}$. We followed the instructions of the Alkaline Phosphatase Detection Kit (Nanjing Jiancheng Bioengineering, China). The absorbance of each well was measured at $520 \mathrm{~nm}$ using a microplate reader (Bio-Rad, USA). The alkaline phosphatase activity in the sample was calculated from the OD value and the protein concentration of the sample.

\section{Alkaline phosphatase staining}

Cells were fixed in 4\% paraformaldehyde for $10 \mathrm{~min}$, washed three times with PBS, and incubated with ALP staining solution (Alkaline phosphatase staining kit, Beyotime, China) for $30 \mathrm{~min}$, and then washed three times with PBS. An Olympus microscope was used to acquire images.

\section{Alizarin red staining}

The cells were fixed in 4\% paraformaldehyde for $10 \mathrm{~min}$, washed three times with PBS, and incubated with Alizarin red staining solution at room temperature for $3 \mathrm{~min}$. Cells were then washed three times with PBS. An Olympus microscope was used to acquire images.

\section{Western blotting}

The total protein samples were extracted according to the kit instructions (Whole Protein Extraction Kit, Beyotime, China). The protein was separated by SDS-PAGE (voltage of $80 / 120 \mathrm{~V}$ ) and transferred to PVDF membranes at a voltage of $60 \mathrm{~V}$ for $3 \mathrm{~h}$. The membranes were blocked with $5 \%$ nonfat dry milk for $1 \mathrm{~h}$ at room temperature, incubated with primary antibody: GAPDH (1:8000, Cell Signaling Technology, USA); $\beta$-Actin (1:1000, Bioworld, China); OPN (1:1000, Abcam, USA); RUNX2 (1:1000, Abcam, USA); FAK (1:500, Cell Signaling Technology, USA); Talin (1:1000, Millipore, USA); Vinculin (1:800, Sigma-Aldrich, USA); Paxillin (1:1000, Becton Dickinson and Company, USA); Zyxin (1:700, Affinity, USA); YAP (1:700, Cell Signaling Technology, USA); P-YAP (1:1000, Cell Signaling Technology, USA) overnight at $4{ }^{\circ} \mathrm{C}$ with gentle shaking, and incubated with appropriate secondary antibodies: horseradish peroxidase-labeled goat anti-rabbit IgG $(\mathrm{H}+\mathrm{L})$ (1:5000, Fdbio Science, China); horseradish peroxidase-labeled goat anti-rabbit IgG $(\mathrm{H}+\mathrm{L})(1: 5000$, Fdbio science, China) at room temperature for $1 \mathrm{~h}$. 
Protein expression was visualized using an exposure instrument (Tanon 5500, Tanon, China). Quantification of western blot data was performed by Gel-pro software.

\section{Statistical analysis}

All experiments were performed in triplicate. Statistical analyses were performed using GraphPad Prism 7.04 software. Results are expressed as means \pm standard deviation (SD). A t-test was used for comparison between the two groups, and ANOVA was used for comparison between multiple groups. $\mathrm{P}<0.05$ was considered to indicate statistically significant results.

\section{Results}

\section{Effect of different actin polymerization states on proliferation of hASCs}

We evaluated the effects of different polymerization states of actin on the proliferation of hASCs. Here, we performed both Ki67 immunofluorescence staining and CCK-8 experiments. The results revealed that the cell proliferation rate in the JAS $(1,5,10$, 20 , and $50 \mathrm{nM}$ ) groups was higher than that in the control; furthermore, it gradually increased with the increasing JAS concentrations, but significantly decreased in the JAS (50 nM) group (Fig. 1a). The CCK-8 result and cell counter assay were consistent with Ki67 results (Fig. 1b, c). These findings suggest that the actin polymerization caused by JAS at the lower concentration range (1-20 nM) promoted cell proliferation, while the high concentrations JAS (50 $\mathrm{nM})$ inhibited it.

\section{hASC apoptosis and its relationship with actin polymerization state}

To investigate the effects of different actin polymerization states on cell apoptosis, we performed the Annexin V-FITC/PI apoptosis assay. Flow cytometry results confirmed that the cell apoptosis rates of the JAS $(1,5,10$, and $20 \mathrm{nM})$ groups were lower than that of the control group, indicating that a high concentration of JAS promoted cell apoptosis. In contrast, the cell apoptosis rate in the JAS $50 \mathrm{nM}$ group was markedly increased $(\mathrm{Q} 2+\mathrm{Q} 3=0.879 \%)$ (Fig. 2a), indicating that a high concentration of JAS promoted cell apoptosis.

\section{The influence of different actin aggregation states on hASC migration}

Previous research has demonstrated that the state of actin filaments can regulate cell movement. To study the effect of different polymerization states of actin on cell migration, we conducted wound healing and transwell experiments, respectively. The wound healing results suggested that the healing speed in the JAS $(1,5,10$, and $20 \mathrm{nM})$ groups was faster than that of the control group, while the JAS (50 nM) group was slower than the control group (Fig. 3a). Transwell results (Fig. 3b) showed that in the JAS (1, 5, 10, and $20 \mathrm{nM}$ ) groups, the number of cells in the lower chamber gradually increased but decreased sharply in the JAS ( $50 \mathrm{nM}$ ) group (which was significantly lower than the control group). Transwell results were generally consistent with the wound healing results. These data demonstrated that actin polymerization induced by a low concentration of JAS is beneficial for increasing cell migration. In contrast, a high concentration of JAS induced actin polymerization and negatively regulated cell migration. 


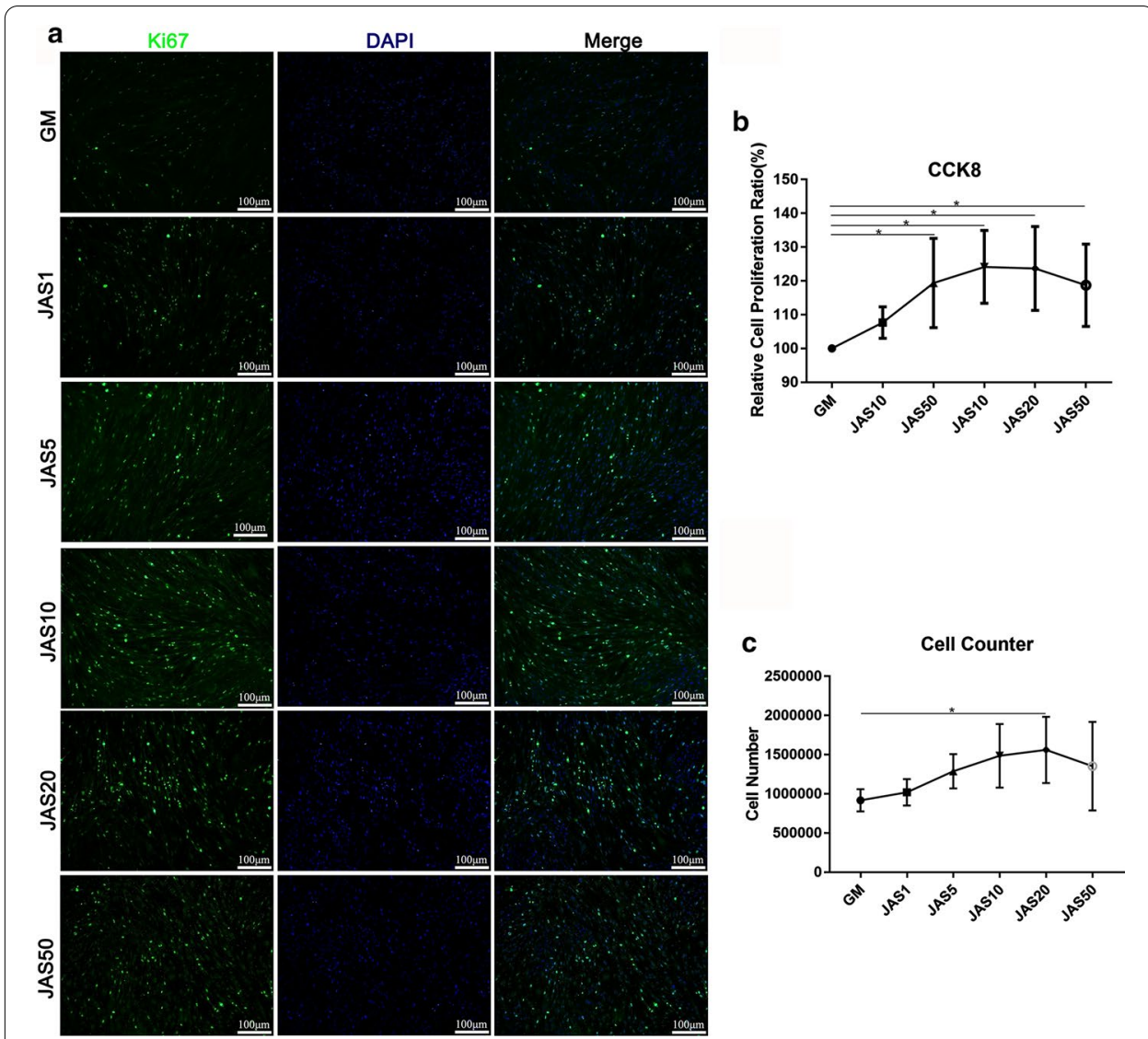

Fig. 1 The effect of different actin polymerization states on the proliferation of hASCs. a Control and JAS-treated hASCs stained with Ki67 (green) at day 7. Quantification of the Ki67 positive rate was performed using ImageJ; $\mathbf{b}$ CCK-8 analysis of hASCs after treatment with JAS for 7 days. Absorbance was measured at $540 \mathrm{~nm}$; (C) Cell number was obtained using a blood cell count plate. Results are expressed as mean \pm SD; ${ }^{*} p<0.05$. Scale bar $=100 \mu \mathrm{m}$

\section{Different actin polymerization states and the maturity of focal adhesions}

Studies have shown that the dynamic changes of integrins and focal adhesions are involved in cell migration. Therefore, we hypothesize that the different polymerization states of actin result in differences in cell migration because actin polymerization affects the maturity of focal adhesions. To confirm this, we used western blot analysis to detect the protein expression level of focal adhesion-related proteins (FAK, vinculin, talin, paxillin, and zyxin). The results showed that $\beta$-actin, FAK, vinculin, talin, and paxillin were all highly expressed in JAS $(1,5,10,20$ and $50 \mathrm{nM})$ groups, but they were highest in the JAS $(20 \mathrm{nM})$ group, and then subsequently decreased in the JAS $(50 \mathrm{nM})$ group. However, interestingly, the expression of $\beta$-actin and zyxin showed a continuous increase in the JAS $(1,5,10,20$, and $50 \mathrm{nM})$ groups, but there was no decrease in the JAS (50 nM) group (Fig. 4a, b, Additional file 1: Figure S1). Therefore, we believe that actin polymerization could influence the maturity of focal adhesions and that there may be a difference between the influence of low and high actin polymerization states. 


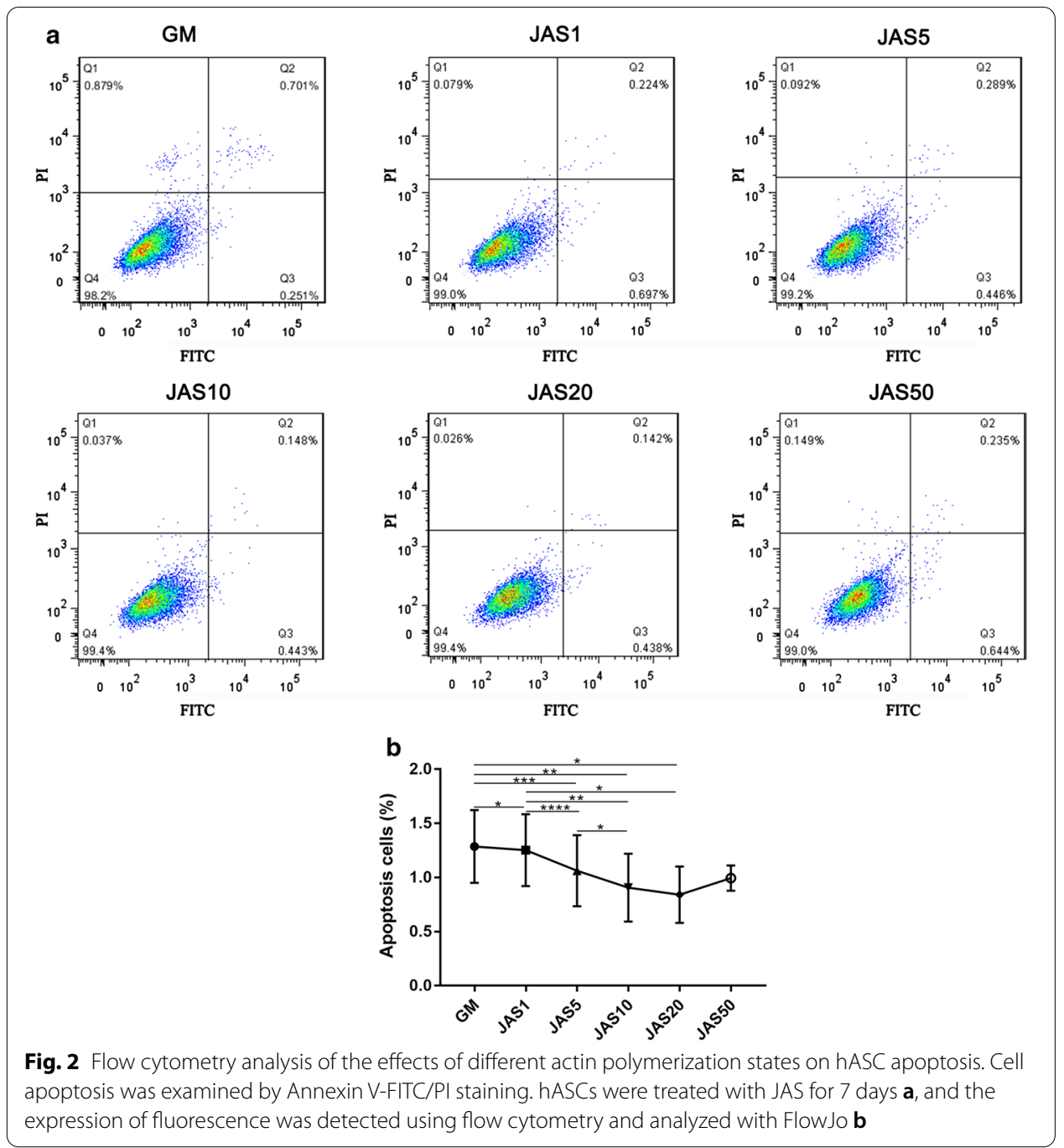

\section{Actin polymerization can promote localization of YAP protein to the cytoplasm}

According to the literature, actin depolymerization can induce the nuclear localization of YAP and regulate gene expression. Therefore, we speculated that different polymerization states of actin might have different effects on the nuclear localization of the YAP protein. To verify this, we used immunofluorescence staining to detect the changes in YAP localization. The results revealed that YAP was mainly concentrated in the nucleus in the control group, but that it began to diffuse into the cytoplasm after cells were treated with JAS. The higher the concentration of JAS, the more apparent was the YAP cytoplasmic localization (Fig. 5a). We also verified this at the protein expression level. The expression of YAP and p-YAP (Ser127) in the JAS (1, 5, 10, 20, and $50 \mathrm{nM}$ ) groups was higher than that in the control group (Fig. 5b, c). As the concentration of JAS increased, the ratio of p-YAP to YAP also increased. These data demonstrated that actin polymerization might potently activate YAP by inducing phosphorylation and cytoplasm localization. 


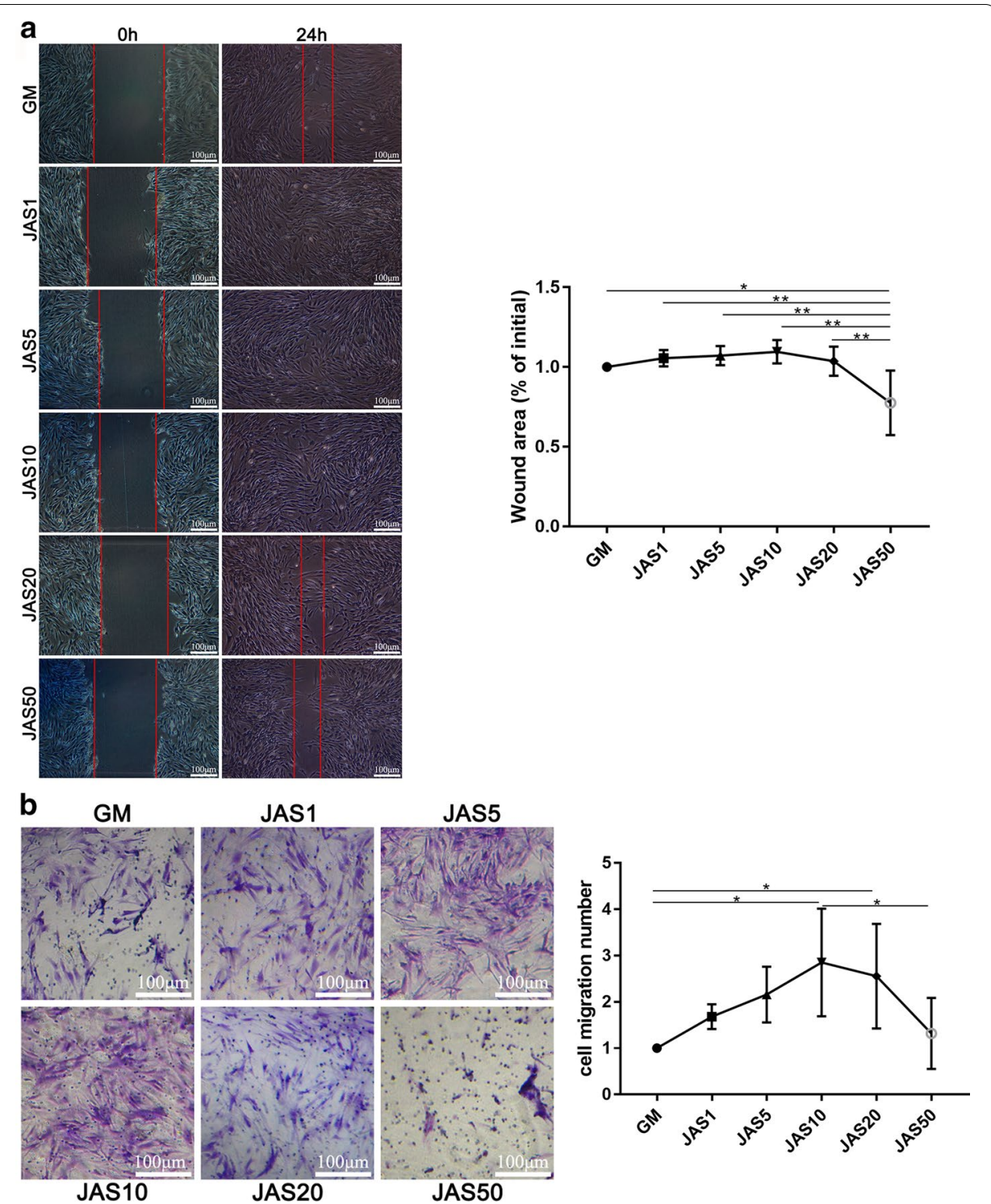

Fig. 3 The influence of different actin aggregation states on hASCs migration. a hASCs were plated on a fibronectin-coated 6-well plate. When cells were completely attached to the plate, confluent monolayers were scratched, and images were captured by microscopy at 0 and $24 \mathrm{~h}$ after the scratch. Quantification of the wound area at 0 and $24 \mathrm{~h}$ was performed using Image J. The wound area was calculated as the percentage of the initial wound area $(0 \mathrm{~h})$. $\mathbf{b}$ We suspended the cells in complete medium without FBS, added $100 \mu \mathrm{L}$ of the cell suspension to the Transwell chamber, and then added medium (20\% FBS) with different concentrations of JAS to the lower chamber. After $24 \mathrm{~h}$, cells were stained with $0.1 \%$ crystal violet, and cell counting was performed using Image J. Results are expressed as mean $\pm S D ;{ }^{*} p<0.05$. Scale $\operatorname{bar}=100 \mu \mathrm{m}$

\section{Effects of different actin polymerization states on ALP activity in hASCs}

Actin polymerization and depolymerization are involved in the regulation of cell differentiation and other biological processes. We believe that JAS may guide the differentiation of cells into osteoblasts. ALP staining and ALP activity assays were performed to explore the effect of actin polymerization on ALP activity in hASCs. The ALP staining results showed that positive staining gradually increased with increasing concentrations 


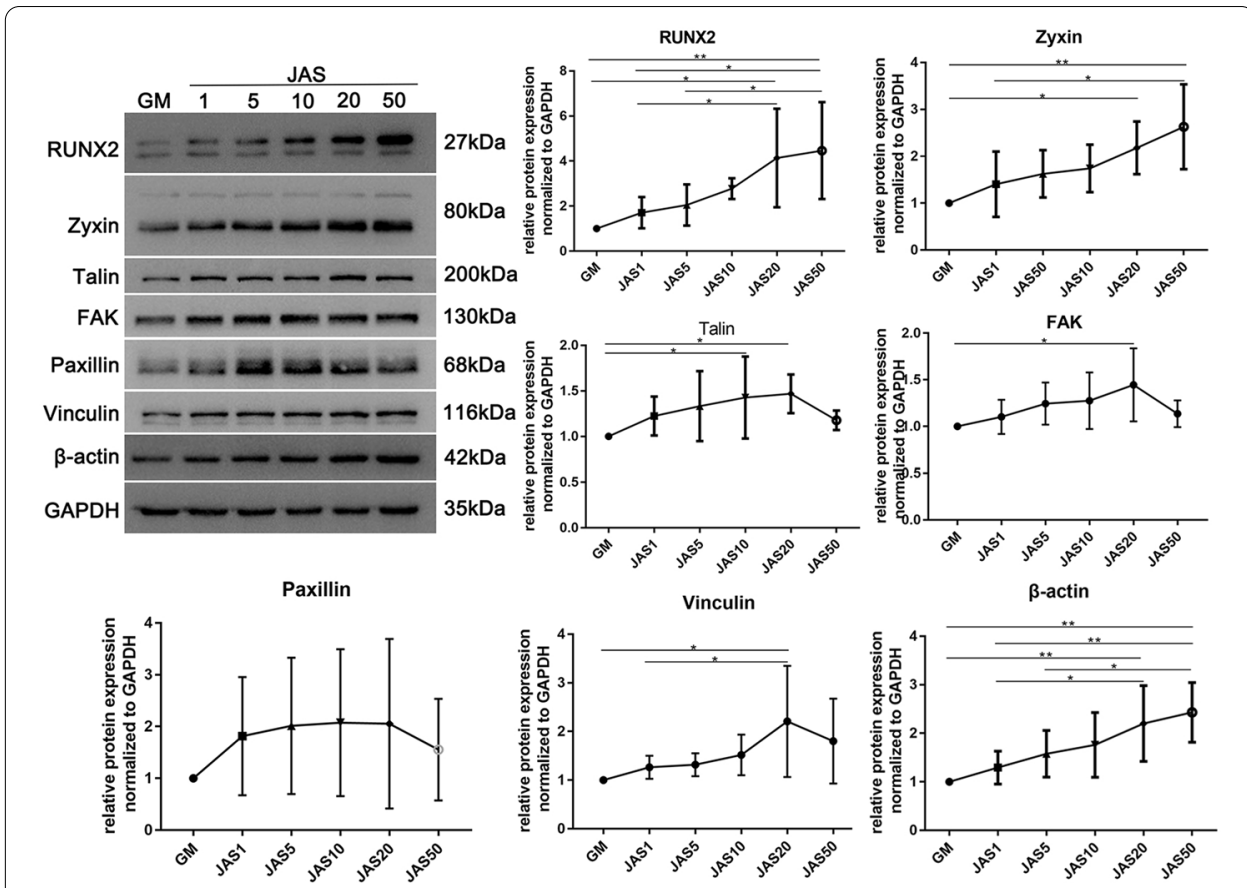

Fig. 4 Different actin polymerization states and the maturity of focal adhesions. Western blot analysis was performed to assess the changes in focal adhesion-related proteins of hASCs cultured in medium containing different concentrations of JAS. Samples were collected after 1 week in culture. Quantification of western blot data was performed by Gel-pro software. The relative expression of these proteins was normalized to that of GAPDH. Results are expressed as mean \pm SD; ${ }^{*} p<0.05$

of JAS (Fig. 6b), from 0 to $20 \mathrm{nM}$; however, at the concentration of $50 \mathrm{nM}$, ALP activity was inhibited (Fig. 6a). The results of the ALP activity assay were approximately consistent with the results of ALP staining. Therefore, our results demonstrate that actin polymerization leads to an increase in ALP activity and content, which can influence cell fate and subsequently direct cells towards an osteogenic lineage.

\section{Actin polymerization state and osteogenic differentiation of hASCs}

The influence of different actin polymerization states on the osteogenic ability of hASCs was investigated by treating the cells with osteogenic induction medium (OS) containing different concentrations of JAS. The alizarin red assay showed that the positive staining in the JAS $(1,5,10,20$ and $50 \mathrm{nM})$ groups was higher than that in the control group and strongest in the JAS ( $50 \mathrm{nM}$ ) group (Fig. 7a). Western blotting results demonstrated that osteogenic differentiation markers, such as OPN and RUNX2, were highly expressed in the JAS $(1,5,10,20$, and $50 \mathrm{nM})$ groups compared with levels in the control group (Fig. 7b). These results suggest that JAS may stimulate the osteogenic differentiation of hASCs in a concentration-dependent manner.

\section{Discussion}

In this study, we used different concentrations of JAS (an actin polymerization agent) to determine the effects of varying actin polymerization states on the osteogenic differentiation of hASCs. These results, including CCK-8, Ki67 immunofluorescence staining, 


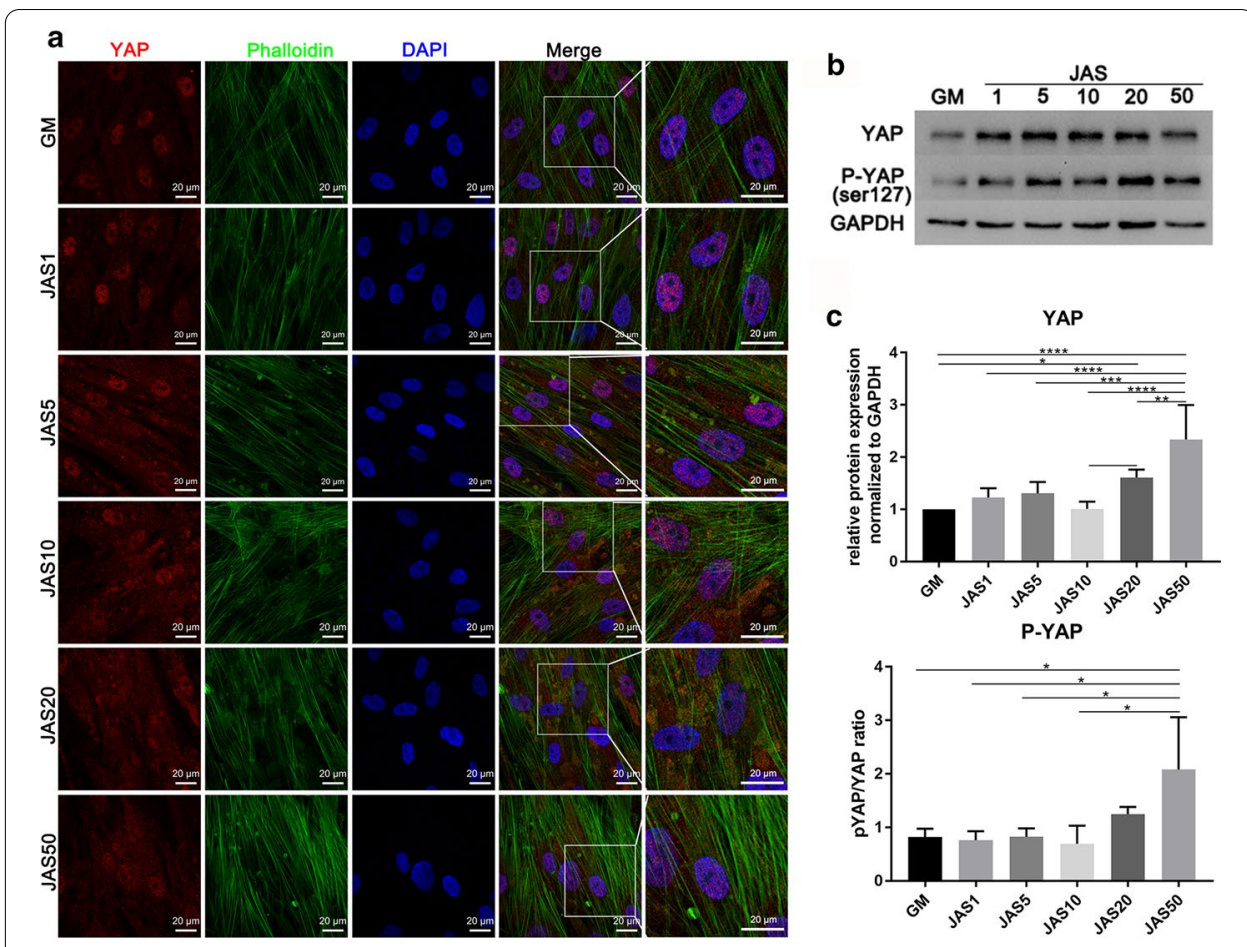

Fig. 5 Actin polymerization can promote localization of YAP protein to the cytoplasm. a Cells were cultured in medium containing different concentrations of JAS. Then, cells were fixed and immunofluorescence stained with anti-YAP antibodies. Fluorescence for YAP and actin is shown in red and green, respectively. Nuclear staining is represented in blue. All images were obtained using a $63 \times$ oil immersion lens on the confocal microscope; $\mathbf{b}$ Western blot analysis for YAP and p-YAP (Ser127) of hASCs cultured in medium containing different concentrations of JAS. Scale bar $=20 \mu \mathrm{m}$

cell apoptosis, wound healing, and the transwell assay, showed that JAS could promote cell proliferation and migration within a specific concentration range. These data indicated that in the JAS $(50 \mathrm{nM})$ group, cell survival rate, cell proliferation, and migration significantly decreased, while the osteogenic differentiation ability increased. Therefore, we concluded that the polymerization of actin might trigger some osteogenesis-related signaling pathways. These results revealed that the low states of actin polymerization promoted cell proliferation and that high states induced cell apoptosis. This may be due, in part, to JAS cytotoxicity at high concentrations, and the subsequent triggering of downstream signaling pathways related to cell apoptosis.

Cell migration depends on the activity of integrins and the maturation of focal adhesions. According to these findings, the different actin polymerization states resulted in varying focal adhesion maturity (Fig. 4a, b). It is noteworthy that the expression level of zyxin did not decrease at JAS ( $50 \mathrm{nM})$, but continued to increase. This trend is consistent with the osteogenic differentiation ability of cells in the OS + JAS (50 nM) group (Fig. 7a, b). Therefore, zyxin, a focal adhesion protein, may have a key role for F-actin to regulate the osteogenic differentiation of cells. In the follow-up research project, we will focus on this key protein (zyxin). Additionally, under the action of a high concentration of JAS (50 nM), the mechanism of the decrease of partial adhesion-related protein expression is still unclear, and further research is urgently needed. 


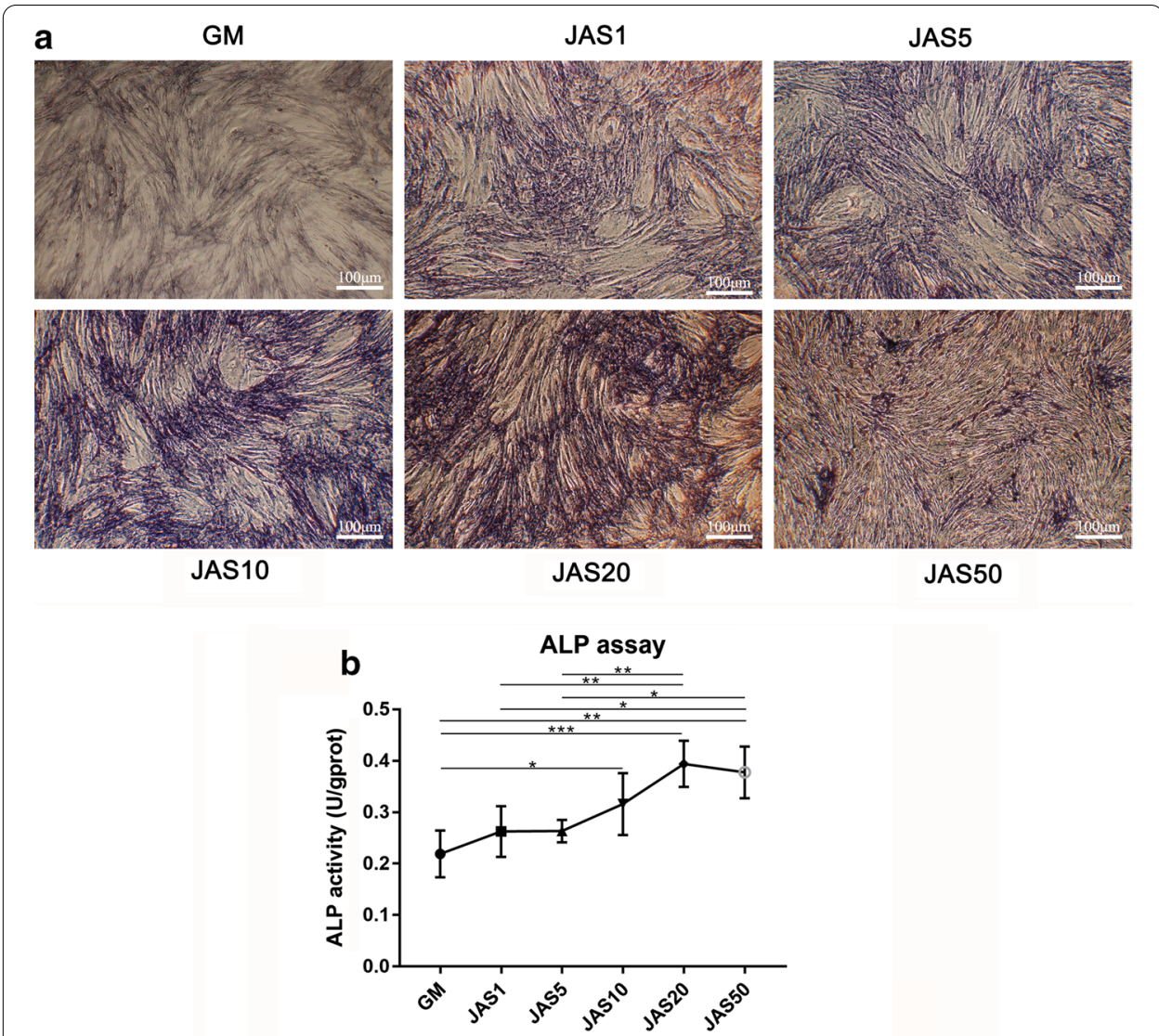

Fig. 6 Effects of different actin polymerization states on ALP activity in hASCs. a ALP staining of hASCs after treatment with JAS for 7 days; $\mathbf{b}$ Analysis of ALP activity of hASCs after treatment with JAS for 7 days. Values are mean $\pm S D ;{ }^{*} p<0.05$. Scale bar $=100 \mu \mathrm{m}$

Osteogenic differentiation of hASCs is a complex process in which multiple genes, proteins, and signaling pathways interact with each other [44-46]. The reorganization of the actin cytoskeleton plays an essential role during stem cell differentiation [42]. Previous work has shown that both the location and polymerization of cellular actin aid in the regulation of differentiation. The formation of actin stress fibers is essential for osteogenesis, while its inhibition stimulates lipogenesis [42]. A study found that actin polymerization and depolymerization are involved in the regulation of cell differentiation and other biological processes [44, 47]. A large number of studies have shown that there is an inverse correlation between actin polymerization and adipogenesis, while there is a direct correlation between actin polymerization and osteogenesis [47]. This result is consistent with our findings; that is, the higher the actin polymerization state, the stronger the ability of osteogenic differentiation. Actin depolymerization increases the levels of phosphorylated p38 and ERK1/2 during adipogenesis, and also increases the gene expression of PPAR $\gamma$ [48]. A similar finding was also reported in another study, which showed that myoblast differentiation and osteoblast differentiation are regulated by the p38 MAPK and ERK1/2 pathways of actin filament remodeling [49].

Integrins form the cell matrix connection of actin, through which ECM substances such as fibronectin are connected to the actin cytoskeleton [50]. It has been proved 


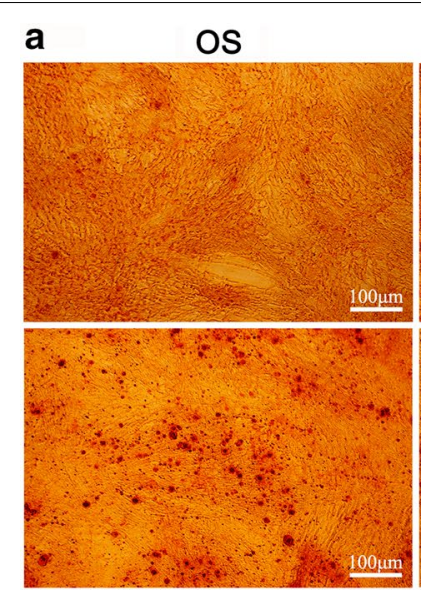

OS+JAS10
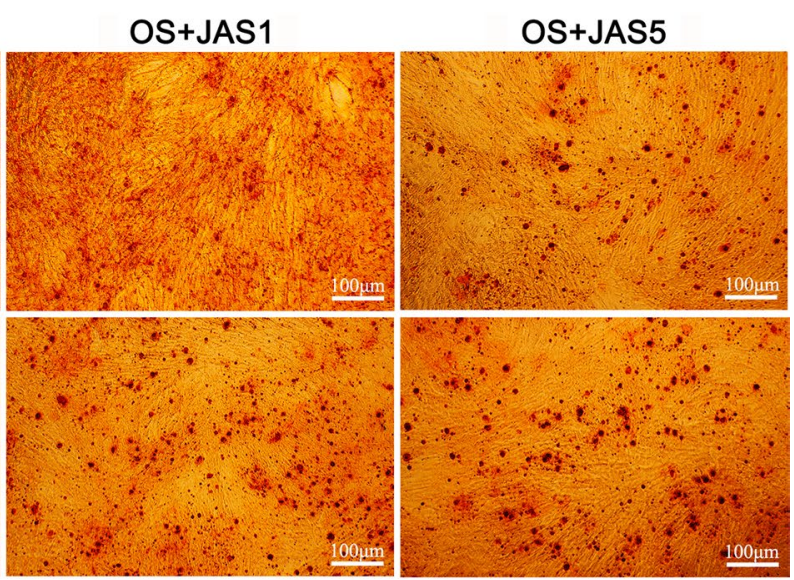

OS+JAS20

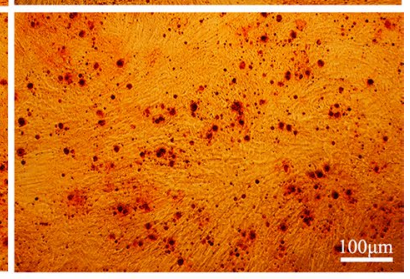

OS+JAS50

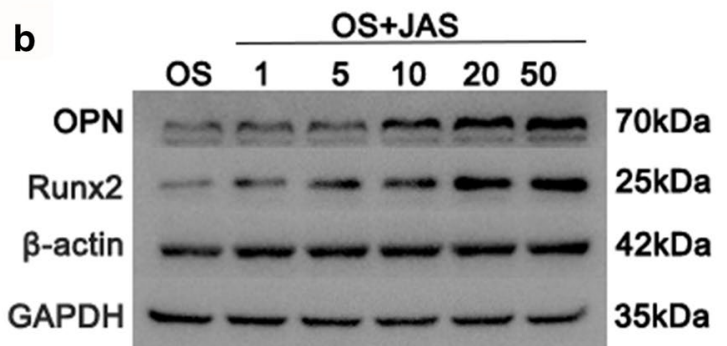

Fig. 7 Actin polymerization state and osteogenic differentiation of hASCs. a Cells were treated with osteogenic induction medium (containing different concentrations of JAS). They were then stained with alizarin red. $\mathbf{b}$ Western blot analysis for osteogenic markers of hASC s cultured in the osteogenic induction medium (containing different concentrations of JAS). Samples were collected after one week in culture. Quantification of western blot data was performed by Gel-pro software. The relative expression of these proteins was normalized to that of GAPDH. Results are expressed as mean \pm SD; ${ }^{*} p<0.05$. Scale bar $=100 \mu \mathrm{m}$

that the cytoskeletal changes necessary for osteogenic formation are integrin-dependent [51-53]. The binding of extracellular components to the cytoskeleton is carried out through the cytoplasmic domain of the integrin that forms the adhesion zone. These adhesion sites are composed of liposomes (protein complexes) that allow mechanical coupling [54-57]. The intracellular actin cytoskeleton senses the cell forces through local adhesome, which leads to the activation of multiple mechanically sensitive pathways, including YAP/TAZ and MKL1 [58, 59]. Integrin-mediated adhesion to ECM is an essential step in determining cell fate during differentiation [60-64]. At the same time, studies have shown that the interaction between cytoskeleton proteins, integrins and mechanical forces can affect cells to change shape, proliferate and even differentiate. It has been found that integrin $\alpha 5$, which is up-regulated during osteogenic differentiation, is an important regulator of osteogenic differentiation. Silencing of integrin $\alpha 5$ eliminated osteogenic differentiation [60].

We know that the actin cytoskeleton is a key determinant of cell shape, which can be explained as the assembly and disassembly of actin filaments [65-67]. Various biological processes such as proliferation and differentiation are affected by cell shape [68-70]. In addition, the actin cytoskeleton-mediated cell morphology changes have been shown to be important for the regulation of MSC lineage commitment and are related to the 
RhoA pathway [19]. Similarly, the ability of cell shape to determine MSC differentiation is reliant on the actin cytoskeleton and the microtubule skeleton [71]. Therefore, we speculate that the different polymerization states of actin cause changes in cell morphology through the Rho pathway, which may have an impact on the osteogenic differentiation of hASCs. At the same time, the modification of the cytoskeleton caused by Rho GTPase has been found to be the main contributor to the differentiation and migration of mesenchymal stem cells (MSC) [19, 72]. Some studies have shown that these kinases (Rho, ROCK, Rac, LIMK) may be regulators of osteoblast differentiation [73]. These signal transduction pathways can play a role not only by changing the cytoskeleton organization of actin, but also through FAK, JNK and p38 MAPK pathways [44, 74]. The differentiation of osteoblasts is affected not only by cell proliferation, but also by cell geometry [71].

Eunjeong et al. reported that YAP1 binds $\beta$-catenin and induces Dkk1, a negative regulator of Wnt signaling, to maintain stemness and prevent osteogenesis [75]. Qiao $\mathrm{Y}$ et al. found that YAP promotes the expression of ARHGAP29 to inhibit the RhoALIMK-cofilin pathway of apoptosis, destabilizing F-actin, promotes actin depolymerization, and reduces the stiffness of the cytoskeleton [76]. In contrast, Pan and colleagues demonstrated the mechanism by which YAP regulates fat bone formation. They found that YAP interacts with $\beta$-catenin to promote osteogenic differentiation and maintain bone homeostasis in the mouse model [77]. A number of studies have demonstrated that alteration of actin dynamics exerts a strong impact on the activity of YAP/TAZ. The various regulatory inputs that determine YAP/TAZ activity are aggregated on the actin cytoskeleton, so induction of filamentous actin (F-actin) bundling by knockdown of F-actin capping or severing proteins promotes nuclear enrichment of YAP/TAZ [78-81]. In contrast, in many cellular environments, treatment of cells with F-actin interfering agents causes retention of YAP/TAZ in the cytoplasm [82, 83]. Since F-actin is known to integrate multiple regulatory signals and participate in various cellular activities, including migration, polarization, and intracellular transmission, Hippo signaling and YAP/ TAZ activity may be finely regulated through a complex interlinked regulatory network. Studies have shown that increased actin polymerization can lead to nuclear accumulation of YAP. Our experiments revealed that actin polymerization may effectively activate YAP by inducing phosphorylation and cytoplasmic localization. We speculate that this may be related to different experimental systems: we use different cells, different growth media, and the chemical drugs we use might be trigger other signaling pathways to regulate YAP. Solving these problems still requires further investigation.

In general, we speculate that: (1) the higher the degree of actin polymerization, the easier it is to form a cell shape that is conducive to osteogenic differentiation through the Rho pathway, and at the same time, the thicker the actin fibers are and the more F-actin there is; (2) in the process of JAS promoting actin polymerization to promote the osteogenic differentiation of adipose stem cells, it is likely that integrin regulates the phosphorylation of focal adhesion components zyxin and p38 MAPK, thereby affecting the access of nuclear YAP to promote bone formation.

This study has several limitations. First, we focused on the two-dimensional hASC culture environment, which does not well simulate the in vivo environments. When stem cells are cultured on a two-dimensional plastic/glass surface, the F-actin of the 
cells is enhanced compared to the cells in the body. Therefore, it will be more interesting to consider the stiffness of the substrate in this study. Secondly, the lack of relevant quantitative experiments is also a shortcoming in this research. In the next step, we will conduct relevant signal pathway research on the subject through experimental methods such as immunofluorescence semi-quantitative analysis, qRT-PCR, gene down-regulation, and gene sequencing, and we will use CytoD as a negative control to further verify this result.

\section{Conclusion}

In summary, in this study, we treated hASCs with different concentrations of JAS to maintain actin in specific polymerization states. We found that increased polymerization of actin promoted osteogenic differentiation, and this may have been achieved by regulating the focal adhesion-related proteins involved in activating YAP. This work forms the basis for further exploration of the mechanisms underlying the osteogenic differentiation of human adipose-derived stem cells.

\section{Abbreviations}

JAS: Jasplakinolide; OS: Osteogenic differentiation medium; hASCs: Human adipose derived stem cells; FBS: Fetal bovine serum; CCK-8: Cell Counting Kit-8; ALP: Alkaline phosphatase.

\section{Supplementary Information}

The online version contains supplementary material available at https://doi.org/10.1186/s11658-021-00259-8.

Additional file 1: Figure S1. Different actin polymerization states and the maturity of focal adhesions.

Acknowledgments

The authors thank Dr. Feng Lu (Southern Medical University, Guangzhou, China) for help in providing human fat tissue.

Authors' contributions

$J \mathrm{O}$ and JD conceived and designed the experiments. BS, RQ, TF, YY, XJ, AUK, ZZ, JZ and KW performed the experiments. $B S, T F$, and $R Q$ analyzed the data. $B S, R Q, T F$, and JD drafted the manuscript. All authors read and approved the final manuscript.

Funding

This study was financially supported by the National Key R\&D Program of China (2017YFC1105000).

Availability of data and materials

All the supporting data can be downloaded.

\section{Declarations}

Ethics approval and consent to publication

All the protocols in this study were approved by the Ethics Committee of Basic Medical Sciences, Southern Medical University (No. NFYKDXJCYXY2016031002, Date: 2016/03/10, Guangdong, China) and performed in accordance with the Declaration of Helsinki.

Consent for publication

Not applicable.

Competing interests

The authors have no competing interests to report.

\section{Author details}

${ }^{1}$ Guangdong Provincial Key Laboratory of Medical Biomechanics and Department of Anatomy, School of Basic Medical Science, Southern Medical University, Guangzhou, China. ${ }^{2}$ Central Laboratory, Southern Medical University, Guangzhou, China. ${ }^{3}$ Department of Foot and Ankle Surgery, Henan Luoyang Orthopedic Hospital, Zhengzhou, China. ${ }^{4}$ Division of Orthopaedics and Traumatology, Department of Orthopaedics, Guangdong Provincial Key Laboratory of Bone and Cartilage Regeneration Medicine, Nanfang Hospital, Southern Medical University, Guangzhou, China. 
Received: 28 January 2021 Accepted: 3 April 2021

Published online: 15 April 2021

\section{References}

1. Chugh P, Paluch EK. The actin cortex at a glance. J Cell Sci. 2018;131:s186254

2. Svitkina T. The actin cytoskeleton and actin-based motility. CSH Perspect Biol. 2018;10:a18267.

3. Peckham M. How myosin organization of the actin cytoskeleton contributes to the cancer phenotype. Biochem Soc Trans. 2016:44:1026-34.

4. Vicente-Manzanares M, Ma X, Adelstein RS, Horwitz AR. Non-muscle myosin II takes centre stage in cell adhesion and migration. Nat Rev Mol Cell Biol. 2009;10:778-90.

5. Pollard TD, Cooper JA. Actin, a central player in cell shape and movement. Science. 2009;326:1208-12.

6. Blanchoin L, Boujemaa-Paterski R, Sykes C, Plastino J. Actin dynamics, architecture, and mechanics in cell motility. Physiol Rev. 2014;94:235-63.

7. Bezanilla M, Gladfelter AS, Kovar DR, Lee W. Cytoskeletal dynamics: a view from the membrane. J Cell Biol. 2015;209:329-37.

8. Yuan B, Zhang R, Hu J, Liu Z, Yang C, Zhang T, et al. WDR1 promotes cell growth and migration and contributes to malignant phenotypes of non-small cell lung cancer through ADF/cofilin-mediated actin dynamics. Int J Biol Sci. 2018;14:1067-80.

9. Izdebska M, Zielińska W, Grzanka D, Gagat M. The role of actin dynamics and actin-binding proteins expression in epithelial-to-mesenchymal transition and its association with cancer progression and evaluation of possible therapeutic targets. Biomed Res Int. 2018;2018:1-13.

10. Stortz JF, Del RM, Singer M, Wilkes JM, Meissner M, Das S. Formin-2 drives polymerisation of actin filaments enabling segregation of apicoplasts and cytokinesis in Plasmodium falciparum. Elife. 2019. https://doi.org/10.7554/eLife. 49030.

11. Rug M, Cyrklaff M, Mikkonen A, Lemgruber L, Kuelzer S, Sanchez CP, et al. Export of virulence proteins by malariainfected erythrocytes involves remodeling of host actin cytoskeleton. Blood. 2014;124:3459-68.

12. Cyrklaff M, Sanchez CP, Frischknecht F, Lanzer M. Host actin remodeling and protection from malaria by hemoglobinopathies. Trends Parasitol. 2012;28:479-85.

13. Seixas Al, Azevedo MM, Paes DFJ, Fernandes D, Mendes PI, Relvas JB. Evolvability of the actin cytoskeleton in oligodendrocytes during central nervous system development and aging. Cell Mol Life Sci. 2019;76:1-11.

14. Matsubayashi Y, Coulson-Gilmer C, Millard TH. Endocytosis-dependent coordination of multiple actin regulators is required for wound healing. J Cell Biol. 2015:210:419-33.

15. Schmit AC, Lambert AM. Microinjected fluorescent phalloidin in vivo reveals the F-actin dynamics and assembly in higher plant mitotic cells. Plant Cell. 1990;2:129-38.

16. Shoji K, Ohashi K, Sampei K, Oikawa M, Mizuno K. Cytochalasin D acts as an inhibitor of the actin-cofilin interaction. Biochem Bioph Res Commun. 2012;424:52-7.

17. Kopecká M, Kawamoto S, Yamaguchi M. Effects of the F-actin inhibitor latrunculin A on the budding yeast Saccharomyces cerevisiae. Microbiology. 2015;161:1348-55.

18. Fujiwara I, Zweifel ME, Courtemanche N, Pollard TD. Latrunculin A accelerates actin filament depolymerization in addition to sequestering actin monomers. Curr Biol. 2018;28:3183-92.

19. McBeath R, Pirone DM, Nelson CM, Bhadriraju K, Chen CS. Cell shape, cytoskeletal tension, and RhoA regulate stem cell lineage commitment. Dev Cell. 2004;6:483-95.

20. Zabriskie TM, Klocke JA, Ireland CM, Marcus AH, Molinski TF, Faulkner DJ, et al. Jaspamide, a modified peptide from a Jaspis sponge, with insecticidal and antifungal activity. J Am Chem Soc. 1986;108:3123-4.

21. Terracciano S, Bruno I, D’Amico E, Bifulco G, Zampella A, Sepe V, et al. Synthetic and pharmacological studies on new simplified analogues of the potent actin-targeting Jaspamide. Bioorgan Med Chem. 2008;16:65808.

22. Terada Y, Simerly C, Schatten G. Microfilament stabilization by jasplakinolide arrests oocyte maturation, cortical granule exocytosis, sperm incorporation cone resorption, and cell-cycle progression, but not DNA replication, during fertilization in mice. Mol Reprod Dev. 2000;56:89-98.

23. Shurety W, Stewart NL, Stow JL. Fluid-phase markers in the basolateral endocytic pathway accumulate in response to the actin assembly-promoting drug Jasplakinolide. Mol Biol Cell. 1998;9:957-75.

24. Fabian I, Halperin D, Lefter S, Mittelman L, Altstock RT, Seaon O, et al. Alteration of actin organization by jaspamide inhibits ruffling, but not phagocytosis or oxidative burst, in HL-60 cells and human monocytes. Blood. 1999:93:3994-4005.

25. Braekman JC, Daloze D, Moussiaux B, Riccio R. Jaspamide from the marine sponge Jaspis johnstoni. J Nat Prod. 1987:50:994-5.

26. Crews P, Manes LV, Boehler M. Jasplakinolide, a cyclodepsipeptide from the marine sponge, Jaspis SP. Tetrahedron Lett. 1986;27:2797-800

27. Bubb MR, Senderowicz AM, Sausville EA, Duncan KL, Korn ED. Jasplakinolide, a cytotoxic natural product, induces actin polymerization and competitively inhibits the binding of phalloidin to F-actin. J Biol Chem. 1994;269:14869.

28. Matthews JB, Smith JA, Hrnjez BJ. Effects of F-actin stabilization or disassembly on epithelial Cl-secretion and Na-K2Cl cotransport. Am J Physiol. 1997;272:C254

29. Van Goor D, Hyland C, Schaefer AW, Forscher P. The role of actin turnover in retrograde actin network flow in neuronal growth cones. PLOS ONE. 2012;7:e30959.

30. Hagiwara A, Tanaka Y, Hikawa R, Morone N, Kusumi A, Kimura H, et al. Submembranous septins as relatively stable components of actin-based membrane skeleton. Cytoskeleton. 2011;68:512-25.

31. Zuk PA, Zhu M, Mizuno H, Huang J, Futrell JW, Katz AJ, et al. Multilineage cells from human adipose tissue: implications for cell-based therapies. Tissue Eng. 2001;7:211-28. 
32. Tholpady SS, Llull R, Ogle RC, Rubin JP, Futrell JW, Katz AJ. Adipose tissue: stem cells and beyond. Clin Plast Surg. 2006;33:55-62.

33. Rodriguez AM, Elabd C, Amri E, Ailhaud G, Dani C. The human adipose tissue is a source of multipotent stem cells. Biochimie. 2005;87:125-8.

34. Gimble JM, Guilak F, Bunnell BA. Clinical and preclinical translation of cell-based therapies using adipose tissuederived cells. Stem Cell Res Ther. 2010;1:19.

35. Mesimäki K, Lindroos B, Törnwall J, Mauno J, Lindqvist C, Kontio R, et al. Novel maxillary reconstruction with ectopic bone formation by GMP adipose stem cells. Int J Oral Max Surg. 2009;38:201-9.

36. Bayati V, Sadeghi Y, Shokrgozar MA, Haghighipour N, Azadmanesh K, Amanzadeh A, et al. The evaluation of cyclic uniaxial strain on myogenic differentiation of adipose-derived stem cells. Tissue Cell. 2011;43:359-66.

37. Stromps J, Paul NE, Rath B, Nourbakhsh M, Bernhagen J, Pallua N. Chondrogenic differentiation of human adiposederived stem cells: a new path in articular cartilage defect management? Biomed Res Int. 2014;2014:1-7.

38. Yilgor Huri P, Cook CA, Hutton DL, Goh BC, Gimble JM, DiGirolamo DJ, et al. Biophysical cues enhance myogenesis of human adipose derived stem/stromal cells. Biochem Biophys Res Commun. 2013;438:180-5.

39. Sheykhhasan M, Qomi RT, Ghiasi M. Fibrin scaffolds designing in order to human adipose-derived mesenchymal stem cells differentiation to chondrocytes in the presence of TGF- $\beta$ 3. Int J Stem Cells. 2015;8:219-27.

40. McIntosh K, Zvonic S, Garrett S, Mitchell JB, Floyd ZE, Hammill L, et al. The immunogenicity of human adiposederived cells: temporal changes in vitro. Stem Cells. 2006;24:1246-53.

41. Bamburg JR, Wiggan OP. ADF/cofilin and actin dynamics in disease. Trends Cell Biol. 2002;12:598-605.

42. Sen B, Uzer G, Samsonraj RM, Xie Z, McGrath C, Styner M, et al. Intranuclear actin structure modulates mesenchymal stem cell differentiation. Stem Cells. 2017;35:1624-35.

43. Du Y, Montoya C, Orrego S, Wei X, Ling J, Lelkes PI, et al. Topographic cues of a novel bilayered scaffold modulate dental pulp stem cells differentiation by regulating YAP signalling through cytoskeleton adjustments. Cell Prolif. 2019. https://doi.org/10.1111/cpr.12676.

44. Ye G, Li C, Xiang X, Chen C, Zhang R, Yang X, et al. Bone morphogenetic protein-9 induces PDLSCs osteogenic differentiation through the ERK and p38 signal pathways. Int J Med Sci. 2014;11:1065-72.

45. Li L, Dong Q, Wang Y, Feng Q, Zhou P, Ou X, et al. Hedgehog signaling is involved in the BMP9-induced osteogenic differentiation of mesenchymal stem cells. Int J Mol Med. 2015;35:1641-50.

46. Gu H, Huang Z, Yin X, Zhang J, Gong L, Chen J, et al. Role of C-Jun N-terminal kinase in the osteogenic and adipogenic differentiation of human adipose-derived mesenchymal stem cells. Exp Cell Res. 2015;339:112-21.

47. Chen L, Hu H, Qiu W, Shi K, Kassem M. Actin depolymerization enhances adipogenic differentiation in human stromal stem cells. Stem Cell Res. 2018;29:76-83.

48. Xu B, Ju Y, Song G. Role of p38, ERK1/2, focal adhesion kinase, RhoA/ROCK and cytoskeleton in the adipogenesis of human mesenchymal stem cells. J Biosci Bioeng. 2014;117:624-31.

49. Sonowal H, Kumar A, Bhattacharyya J, Gogoi PK, Jaganathan BG. Inhibition of actin polymerization decreases osteogeneic differentiation of mesenchymal stem cells through p38 MAPK pathway. J Biomed Sci. 2013;20:71.

50. Kundu AK, Khatiwala CB, Putnam AJ. Extracellular matrix remodeling, integrin expression, and downstream signaling pathways influence the osteogenic differentiation of mesenchymal stem cells on poly(lactide-co-glycolide) substrates. Tissue Eng Part A. 2009;15:273-83.

51. Shih YR, Tseng KF, Lai HY, Lin CH, Lee OK. Matrix stiffness regulation of integrin-mediated mechanotransduction during osteogenic differentiation of human mesenchymal stem cells. J Bone Miner Res. 2011;26:730-8.

52. Treiser MD, Yang EH, Gordonov S, Cohen DM, Androulakis IP, Kohn J, et al. Cytoskeleton-based forecasting of stem cell lineage fates. Proc Natl Acad Sci U S A. 2010;107:610-5.

53. Salasznyk RM, Klees RF, Williams WA, Boskey A, Plopper GE. Focal adhesion kinase signaling pathways regulate the osteogenic differentiation of human mesenchymal stem cells. Exp Cell Res. 2007;313:22-37.

54. Maître JL, Berthoumieux H, Krens SF, Salbreux G, Jülicher F, Paluch E, et al. Adhesion functions in cell sorting by mechanically coupling the cortices of adhering cells. Science. 2012;338:253-6.

55. Iskratsch T, Wolfenson H, Sheetz MP. Appreciating force and shape-the rise of mechanotransduction in cell biology. Nat Rev Mol Cell Biol. 2014;15:825-33.

56. Bershadsky AD, Balaban NQ, Geiger B. Adhesion-dependent cell mechanosensitivity. Annu Rev Cell Dev Biol. 2003;19:677-95.

57. Sastry SK, Burridge K. Focal adhesions: a nexus for intracellular signaling and cytoskeletal dynamics. Exp Cell Res. 2000;261:25-36.

58. Speight P, Kofler M, Szászi K, Kapus A. Context-dependent switch in chemo/mechanotransduction via multilevel crosstalk among cytoskeleton-regulated MRTF and TAZ and TGF $\beta$-regulated Smad3. Nat Commun. 2016;7:11642.

59. Low BC, Pan CQ, Shivashankar GV, Bershadsky A, Sudol M, Sheetz M. YAP/TAZ as mechanosensors and mechanotransducers in regulating organ size and tumor growth. Febs Lett. 2014;588:2663-70.

60. Hamidouche Z, Fromigué O, Ringe J, Häupl T, Vaudin P, Pagès JC, et al. Priming integrin alpha5 promotes human mesenchymal stromal cell osteoblast differentiation and osteogenesis. Proc Natl Acad Sci U S A. 2009;106:18587-91.

61. Born AK, Rottmar M, Lischer S, Pleskova M, Bruinink A, Maniura-Weber K. Correlating cell architecture with osteogenesis: first steps towards live single cell monitoring. Eur Cell Mater. 2009;18:49-60, 61-2, 60.

62. Osugi Y, Fumoto K, Kikuchi A. CKAP4 regulates cell migration via the interaction with and recycling of integrin. Mo Cell Biol. 2019. https://doi.org/10.1128/MCB.00073-19.

63. Hight-Warburton W, Parsons M. Regulation of cell migration by a4 and a9 integrins. Biochem J. 2019;476:705-18.

64. De Pascalis C, Etienne-Manneville S. Single and collective cell migration: the mechanics of adhesions. Mol Biol Cell. 2017:28:1833-46.

65. Shao D, Levine H, Rappel WJ. Coupling actin flow, adhesion, and morphology in a computational cell motility model. Proc Natl Acad Sci U S A. 2012;109:6851-6.

66. Ujihara Y, Nakamura M, Miyazaki H, Wada S. Segmentation and morphometric analysis of cells from fluorescence microscopy images of cytoskeletons. Comput Math Methods Med. 2013;2013:381356. 
67. Müller P, Langenbach A, Kaminski A, Rychly J. Modulating the actin cytoskeleton affects mechanically induced signal transduction and differentiation in mesenchymal stem cells. PLoS ONE. 2013;8:e71283.

68. Chen CS, Mrksich M, Huang S, Whitesides GM, Ingber DE. Geometric control of cell life and death. Science. 1997;276:1425-8.

69. Watt FM, Jordan PW, O'Neill CH. Cell shape controls terminal differentiation of human epidermal keratinocytes. Proc Natl Acad Sci U S A. 1988;85:5576-80.

70. Roskelley CD, Desprez PY, Bissell MJ. Extracellular matrix-dependent tissue-specific gene expression in mammary epithelial cells requires both physical and biochemical signal transduction. Proc Natl Acad Sci U S A. 1994;91:12378-82.

71. Kilian KA, Bugarija B, Lahn BT, Mrksich M. Geometric cues for directing the differentiation of mesenchymal stem cells. Proc Natl Acad Sci U S A. 2010;107:4872-7.

72. Jaganathan BG, Ruester B, Dressel L, Stein S, Grez M, Seifried E, et al. Rho inhibition induces migration of mesenchymal stromal cells. Stem Cells. 2007;25:1966-74.

73. Prowse PD, Elliott CG, Hutter J, Hamilton DW. Inhibition of Rac and ROCK signalling influence osteoblast adhesion, differentiation and mineralization on titanium topographies. PLoS ONE. 2013;8:e58898.

74. Chen L, Shi K, Frary CE, Ditzel N, Hu H, Qiu W, et al. Inhibiting actin depolymerization enhances osteoblast differentiation and bone formation in human stromal stem cells. Stem Cell Res. 2015;15:281-9.

75. Seo E, Basu-Roy U, Gunaratne PH, Coarfa C, Lim DS, Basilico C, et al. SOX2 regulates YAP1 to maintain stemness and determine cell fate in the osteo-adipo lineage. Cell Rep. 2013;3:2075-87.

76. Qiao Y, Chen J, Lim YB, Finch-Edmondson ML, Seshachalam VP, Qin L, et al. YAP regulates actin dynamics through ARHGAP29 and promotes metastasis. Cell Rep. 2017;19:1495-502.

77. Pan JX, Xiong L, Zhao K, Zeng P, Wang B, Tang FL, et al. YAP promotes osteogenesis and suppresses adipogenic differentiation by regulating $\beta$-catenin signaling. Bone Res. 2018;6:18.

78. Yu FX, Guan KL. The Hippo pathway: regulators and regulations. Genes Dev. 2013;27:355-71.

79. Matsui Y, Lai ZC. Mutual regulation between Hippo signaling and actin cytoskeleton. Protein Cell. 2013;4:904-10.

80. Aragona M, Panciera T, Manfrin A, Giulitti S, Michielin F, Elvassore N, et al. A mechanical checkpoint controls multicellular growth through YAP/TAZ regulation by actin-processing factors. Cell. 2013;154:1047-59.

81. Gao J, He L, Zhou L, Jing Y, Wang F, Shi Y, et al. Mechanical force regulation of YAP by F-actin and GPCR revealed by super-resolution imaging. Nanoscale. 2020;12:2703-14.

82. Zhao B, Li L, Wang L, Wang CY, Yu J, Guan KL. Cell detachment activates the Hippo pathway via cytoskeleton reorganization to induce anoikis. Genes Dev. 2012;26:54-68

83. Wada K, Itoga K, Okano T, Yonemura S, Sasaki H. Hippo pathway regulation by cell morphology and stress fibers. Development. 2011;138:3907-14.

\section{Publisher's Note}

Springer Nature remains neutral with regard to jurisdictional claims in published maps and institutional affiliations.

- fast, convenient online submission

- thorough peer review by experienced researchers in your field

- rapid publication on acceptance

- support for research data, including large and complex data types

- gold Open Access which fosters wider collaboration and increased citations

- maximum visibility for your research: over $100 \mathrm{M}$ website views per year

At BMC, research is always in progress.

Learn more biomedcentral.com/submissions 